\title{
Extracellular vesicles from senescent hepatic stellate cells promote cell viability of hepatoma cells through increasing EGF secretion from differentiated THP-1 cells
}

\author{
YURI MIYAZOE, SATOSHI MIUMA, HISAMITSU MIYAAKI, YASUKO KANDA, SUGURU NAKASHIKI, \\ RYU SASAKI, MASAFUMI HARAGUCHI, HIDETAKA SHIBATA, TAKUYA HONDA, \\ NAOTA TAURA and KAZUHIKO NAKAO \\ Department of Gastroenterology and Hepatology, \\ Nagasaki University Graduate School of Biomedical Sciences, Nagasaki 852-8501, Japan \\ Received May 8, 2019; Accepted January 17, 2020
}

DOI: $10.3892 /$ br.2020.1279

\begin{abstract}
Since the discovery of the senescence-associated secretory phenotype, the role of senescent hepatic stellate cells (HSCs) in hepatocellular carcinoma (HCC) development has gained increasing attention. Similar to cytokines, extracellular vesicles (EVs) are essential for intercellular communication. However, the function of EVs derived from senescent HSCs in HCC progression has not been extensively studied. The aims of the present study were to characterize the EVs derived from senescent HSCs and determine their role in the tumor microenvironment. Cellular senescence was induced in human hepatic stellate cells (HHSteCs) with various concentrations of etoposide. Induction was confirmed using EdU staining and 53BP1 and p21 immunostaining. EVs were isolated by ultracentrifugation and analyzed by nanoparticle tracking analysis. Multiplex immunoassays were used to compare the levels of growth factors secreted from hepatoma cell lines and macrophage cells pretreated with EVs derived from senescent HHSteCs (senescent EVs) with those pretreated with EVs derived from normal cultured HHSteCs (normal EVs). Treatment with $25 \mu \mathrm{M}$ etoposide for 3 days was the most effective at inducing senescence in HHSteCs. This finding was confirmed by induction of irreversible cell-cycle arrest, upregulation of 53BP1 and p21 expression, and increased SA- $\beta$-gal staining. Senescent HHSteCs released increased quantities of EV particles compared with normally cultured HHSteCs. Multiplex analysis revealed that there
\end{abstract}

Correspondence to: Dr Satoshi Miuma, Department of Gastroenterology and Hepatology, Nagasaki University Graduate School of Biomedical Sciences, 1-7-1 Sakamoto, Nagasaki 852-8501, Japan

E-mail: miuma1002@gmail.com

Key words: epidermal growth factor, etoposide, extracellular vesicles, hepatic stellate cells, hepatocellular carcinoma, senescence-associated secretory phenotype was no difference between hepatoma cell lines treated with normal EVs and those treated with senescent EVs in growth factor secretion. In contrast, the secretion of epidermal growth factor (EGF) was increased by macrophage cells treated with senescent EVs compared with those treated with normal EVs. Furthermore, senescent EVs did not affect the viability of hepatoma cells but increased the viability of hepatoma cells co-cultured with macrophage cells. In conclusion, the release of EVs from senescent HSCs was higher compared with normal HSCs. Furthermore, senescent EVs promoted HCC development by upregulating EGF secretion from macrophages.

\section{Introduction}

Hepatocellular carcinoma (HCC) is the sixth most common type of cancer and the third leading cause of cancer-associated death in the world (1). HCC development is based on cirrhosis, and the number of patients is expected to increase in the future (1-3). Advances in research regarding the underlying biology and pathophysiology of $\mathrm{HCC}$ is required to develop effective means of diagnosis and improved treatments for HCC. The surrounding tumor microenvironment has a notable influence on the development of HCC and is a target of novel cancer therapies $(4,5)$. However, elucidating the underlying mechanisms by which the tumor microenvironment promotes HCC may assist in the development of improved therapeutics $(6,7)$. The HCC tumor microenvironment consists primarily of the surrounding blood vessels, non-parenchymal cells, including fibroblasts, myofibroblasts, macrophages, lymphocytes and sinusoidal endothelial cells, and extracellular proteins such as cytokines and chemokines secreted by these cells $(8,9)$. Hepatic stellate cells (HSCs) are the most abundant type of non-parenchymal cell present in the tumor microenvironment of HCC. They participate in modeling the tumor environment through promoting the transdifferentiation of myofibroblast-like cells, which in turn induces liver fibrosis (10-12). Emerging evidence has shown that tumor-associated macrophages (TAMs) around the tumor lesion facilitate tumor growth (13). TAMs serve important roles in tumor development and have attracted considerable attention as components of the tumor microenvironment (14-16). 
Most HCCs develop as a consequence of progression of liver fibrosis $(17,18)$. HSC activation promotes liver fibrosis through the extracellular production of proteins, such as transforming growth factor- $\beta$, tumor necrosis factor- $\alpha$ and interleukin-6 $(19,20)$; therefore, numerous studies have focused on HSC activation $(21,22)$. Based on this, Yoshimoto et al (23) proposed that senescent HSCs contribute to the development of HCC through the expression of proinflammatory cytokines associated with the senescence-associated secretory phenotype (SASP). Cellular senescence is thought to be a defense mechanism against tumor progression, but under certain circumstances may eventually promote tumor development. However, to the best of our knowledge, the means by which senescent HSCs contribute to the HCC tumor microenvironment has not been studied.

Extracellular vesicles (EVs) and cytokines, participate in extracellular communication in the tumor microenvironment (24). EVs are classified as exosomes (40-100 nm), microvesicles (100-1,000 nm) or apoptotic bodies (1-5 $\mu \mathrm{m})(25-27)$. The contents of EVs vary depending on the condition of the cells and therefore exert differing biological effects (25-29). Senescent HSCs promote HCC development via pro-inflammatory cytokines induced by the SASP (23). However, whether EVs derived from senescent HSCs inhibit or promote HCC development remains unknown. To attain a more comprehensive understanding of the HCC tumor microenvironment, it is necessary to assess the impact that EVs derived from senescent HSCs have on HCC. The aim of the present study was to elucidate the effects of EVs derived from senescent HSCs on the HCC tumor microenvironment. The characteristics of EVs derived from senescent HSCs and their influence on growth factor secretion from hepatoma cells and macrophages were assessed.

\section{Materials and methods}

Cell culture and reagents. Human hepatic stellate cells (HHSteCs) were obtained from SteCM; ScienCell Research Laboratories and maintained in stellate cell medium (ScienCell Research Laboratories) supplemented with 2\% FBS, $1 \%$ penicillin/streptomycin solution (ScienCell Research Laboratories) and $1 \%$ stellate cell growth supplement (ScienCell Research Laboratories). The human HCC cell lines Hep3B and Huh7 (American Type Culture Collection) were maintained in DMEM (Wako Pure Chemical Industries Ltd.) supplemented with $10 \%$ FBS and 1\% PenStrep (Thermo Fisher Scientific, Inc.). The human monocytic leukemia cell line THP-1 (American Type Culture Collection) was cultured in RPMI-1640 medium (Wako Pure Chemical Industries Ltd.) supplemented with 10\% FBS and 1\% PenStrep (Thermo Fisher Scientific, Inc.). All cells were maintained in a humidified incubator with $5 \% \mathrm{CO}_{2}$ at $37^{\circ} \mathrm{C}$. THP-1 cells were induced to differentiate by treating them with $10 \mathrm{mg} \mathrm{ml}^{-1}$ phorbol-12-myristate-13-acetate (Sigma-Aldrich; Merck KGaA) for 3 days. Etoposide (ETP) was purchased from Santa Cruz Biotechnology, Inc. Erlotinib hydrochloride was purchased from Sigma-Aldrich (Merck KGaA).

Immunofluorescence assays, EdU staining and SA- $\beta$-gal staining. Cellular senescence was induced by ETP treatment and confirmed by observing p21 and 53BP1 expression in HHSteCs using immunofluorescence assays. A total of $5 \times 10^{4}$ HHSteCs were mounted on four-chamber slides (Lab-Tek II; Thermo Fisher Scientific, Inc.) and treated with various concentrations of ETP for 3 days. Subsequently, cells were fixed with $4 \%$ paraformaldehyde for $30 \mathrm{~min}$ at room temperature, permeabilized with ice-cold $70 \%$ ethanol and blocked in $1 \% \mathrm{BSA}$ for $1 \mathrm{~h}$ at room temperature. Primary antisera, 1:200 rabbit anti-p21 (cat.no. 29475; Cell Signaling Technology, Inc.) or 1:200 rabbit anti-53BP1 (cat. no. IHC-00001; Bethyl Laboratories, Inc.) were added and the cells were incubated for $1 \mathrm{~h}$ at $20-25^{\circ} \mathrm{C}$. After washing the cells with PBS, secondary antisera (AlexaFluor 488-conjugated donkey anti-rabbit IgG; 1:1,000; cat. no. A11008; Molecular Probes; Thermo Fisher Scientific, Inc.) was added to the cells and incubated for $1 \mathrm{~h}$ at room temperature. The slides were washed, and coverslips were mounted with DAPI Fluoromount-G (SouthernBiotech). The uptake of EdU was observed in the HHSteCs treated with ETP for 3 days, and for cells left to recover, for another 3 days in normal medium following treatment. EdU staining of the HHSteCs was performed using a Click-iT EdU AlexaFluor 594 imaging kit (cat. no. C10339; Thermo Fisher Scientific, Inc.) for $4 \mathrm{~h}$ according to the manufacturer's protocol. Images were acquired using a Keyence All-in-One fluorescence microscope (Keyence Corporation) at x100 magnification. SA- $\beta$-gal staining was performed using a Senescence $\beta$-Galactosidase Staining kit (Cell Signaling Technology, Inc.) according to the manufacturer's protocol. All assays were performed at least in duplicate.

Extraction and quantification of EVs derived from HHSteCs. To collect EVs, $2.5 \times 10^{5} \mathrm{HHSteCs}$ either untreated or pretreated with ETP were seeded in a $100-\mathrm{mm}$ dish and grown in medium containing exo-free FBS (System Biosciences) for 7-10 days. The medium was collected and centrifuged at $300 \mathrm{x} \mathrm{g}$ for $10 \mathrm{~min}$ and at $16,500 \mathrm{x} \mathrm{g}$ for $20 \mathrm{~min}$ at $4^{\circ} \mathrm{C}$ to remove cells and debris, respectively. After filtration with a $220-\mathrm{nm}$ filter, the supernatant was ultra-centrifuged at $150,000 \mathrm{x}$ g for $120 \mathrm{~min}$ at $4^{\circ} \mathrm{C}$. The EV pellet was washed and resuspended in PBS and ultra-centrifuged at $150,000 \mathrm{x}$ g for $120 \mathrm{~min}$ at $4^{\circ} \mathrm{C}$. EVs derived from normal cultured HHSteCs and from senescent HHSteCs were termed 'normal EVs' and 'senescent EVs', respectively. To measure the particle size and number of normal and senescent EVs, 7.5x10 $0^{5}$ HHSteCs were seeded in a 60-mm dish and grown for 3 days. The EVs were extracted in the same manner as described above and quantified using nanoparticle tracking analysis (NanoSight; Malvern Panalytical).

Analysis of EV incorporation into hepatoma cells and macrophage cells. To examine the incorporation of EVs into hepatoma cells and macrophages, Hep3B and THP-1 cells were treated with EVs labeled with PKH67. EVs were labeled using a PKH67 Green Fluorescent Cell Linker Mini kit (cat. no. MIN167-1KT; Sigma-Aldrich; Merck KGaA) for general cell membrane labeling according to the manufacturer's protocol. Labeled EVs were pelleted by ultracentrifugation twice at $150,000 \mathrm{x} g$ for $120 \mathrm{~min}$ at $4^{\circ} \mathrm{C}$ to remove excess dye. Subsequently, $5 \times 10^{4}$ Hep3B and differentiated THP-1 cells each were seeded on four-chamber slides and treated with $2 \times 10^{7}$ labeled EVs daily for 3 days. PKH67 expression was observed under a Keyence 
All-in-One fluorescence microscope at x100 magnification. In addition to the unlabeled EVs, PBS without EVs based on the same procedure previously described (to confirm the absence of residual PKH67) was prepared as a negative control. These experiments were performed in at least duplicate.

Quantification of secreted growth factors. Comprehensive quantification of growth factors secreted by the cells treated with EVs was performed using multiplex immunoassays. A total of $5 \times 10^{5}$ Hep3B, Huh7 and differentiated THP-1 cells each were seeded in a 60-mm culture dish and treated with $2 \times 10^{8} \mathrm{EV}$ particles daily for 3 days and the supernatant was subsequently collected. Growth factors were measured using multiplex immunoassays with a Growth Factor 11-Plex Human ProcartaPlex panel (Invitrogen; Thermo Fisher Scientific, Inc.). Epidermal growth factor (EGF) was quantified in the supernatant with a human EGF ELISA kit (cat. no. DEG00; R\&D Systems, Inc.) according to manufacturer's protocol.

$R N A$ extraction and reverse transcription-quantitative $(R T-q) P C R$. EGF mRNA expression in differentiated THP-1 cells was analyzed using RT-qPCR. The total RNA was extracted from cells using SuperScript III First-strand Synthesis system (cat. no. 18080051 Thermo Fisher Scientific, Inc.), and cDNA synthesis was performed for $15 \mathrm{~min}$ at $42^{\circ} \mathrm{C}$ using $1 \mu \mathrm{g}$ total RNA as a template, RT primer and QuantiTect reverse transcriptase (Superscript III; Thermo Fisher Scientific, Inc.). EGF primers were purchased from Takara Bio, Inc. (cat. no. HA159157) but the sequences of the primers were not disclosed. For qPCR, per a reaction, 1 U LightCycler SYBR Green I Master mix (Roche Diagnostics) was used and the cycling conditions were as follows: Pre-incubation for $5 \mathrm{~min}$ at $95^{\circ} \mathrm{C}$; followed by 30 cycles of $10 \mathrm{sec}$ at $95^{\circ} \mathrm{C}, 10 \mathrm{sec}$ at $60^{\circ} \mathrm{C}$ and $10 \mathrm{sec}$ at $72^{\circ} \mathrm{C}$. GAPDH was used as the endogenous control. The sequences of the GAPDH primers were: Forward, 5'-AGCCACATCGCTCAGACAC-3' and reverse, 5'-GCC CAATACGACCAAATCC-3'. Gene expression was calculated using the $2^{-\triangle \Delta C q}$ method (30). PCR was performed in triplicate.

Cell viability assays. To evaluate the impact of EVs on proliferation of hepatoma cells, the viability of Hep3B cells treated with EVs was determined using an MTS assay. A total of $2.5 \times 10^{3}$ Hep3B cells were seeded in a 96-well plate and treated with either $1 \times 10^{6}$ or $3 \times 10^{6} \mathrm{EVs}$ daily for 3 days either with or without differentiated THP-1 cells. Subsequently, $20 \mu$ l CellTiter96 ${ }^{\circledR}$ AQueous One Solution Reagent (Promega Corporation) was added to each well. Following incubation for $2 \mathrm{~h}$ at $37^{\circ} \mathrm{C}$, the reaction was measured using an automated plate reader (Bio-Rad Laboratories, Inc.) at $490 \mathrm{~nm}$. To evaluate the concentration of erlotinib that could be used whilst maintaining the viability of hepatoma cells, MTS assays were used. A total of $2.5 \times 10^{3}$ Hep3B cells were seeded in a 96-well plate and treated with various concentrations of erlotinib for 3 days. MTS assays were performed in at least duplicate.

Statistical analysis. Data are presented as the mean \pm standard deviation of the mean. Multiple comparisons were performed using an ANOVA with a post-hoc Tukey's test. $\mathrm{P}<0.05$ was considered to indicate a statistically significant difference. For multiplex immunoassays which were used as a comprehensive quantification of growth factors, $>2$-fold difference in secretion was used as the threshold of significance.

\section{Results}

Induction of senescence in HHSteCs with ETP treatment. HHSteCs were treated with 2.5, 5.0, 25, or $50 \mu \mathrm{M}$ ETP for 3 days. Alterations in morphology were observed in the treated HHSteCs compared with the untreated cells. HHSteCs treated with $50 \mu \mathrm{M}$ ETP died within 3 days and the number of cells was notably decreased (Fig. S1). For the HHSteCs treated with 2.5, 5.0 and $25 \mu \mathrm{M}$ ETP, the expression of p21 (a cell cycle arrest marker), 53BP1 (a DNA damage marker) foci and the uptake of EdU were examined. p21 expression and 53BP1 foci were significantly increased at all three ETP concentrations (Fig. 1A and B), whereas EdU uptake decreased (Fig. 1C) compared with the control. To confirm induction of irreversible cell cycle arrest associated with senescence, HHSteCs treated with each of the ETP concentrations were grown in fresh ETP-free recovery medium for 3 days. EdU uptake was still reduced in HHSteCs treated with $25 \mu \mathrm{M}$ ETP (Fig. 1D). In contrast, EdU uptake slightly recovered in HHSteCs treated with 2.5 and 5.0 $\mu \mathrm{M}$ ETP. Therefore, 2.5 and 5.0 $\mu \mathrm{M}$ ETP were inadequate for the induction of senescence in HHSteCs. Finally, induction of senescence was confirmed in HHSteCs treated with $25 \mu \mathrm{M}$ ETP using SA- $\beta$-gal staining (Fig. 1E). The proliferation of HHSteCs treated with $25 \mu \mathrm{M}$ ETP for 3 days was reduced in the recovery medium (Fig. 1F). Thus, $25 \mu \mathrm{M}$ ETP was used for all subsequent experiments for the induction of senescence in HHSteCs.

Particle size and number of senescent HHSteC-derived EVs. After extracting the EVs by ultracentrifugation, the particle size and number of senescent EVs were compared to those of normal EVs using nanoparticle tracking analysis. The size of particles were largely $\sim 120 \mathrm{~nm}$, although some particles were $\sim 200 \mathrm{~nm}$ in size. Therefore, it was considered that the extracted EVs were primarily composed of exosomes with a small number of other small vesicles $(25,26)$. The median particle size was $126 \mathrm{~nm}$ for normal EVs and $120 \mathrm{~nm}$ for senescent EVs (Fig. 2A) and this difference was not significant. To determine the number of EV particles released per HHSteC, the area under the curve (AUC) was calculated using a cell proliferation curve. The cumulative number of EVs in the culture medium was associated with the cumulative number of HHSteCs, although the quantities differed notably between the normal and senescent HHSteC cultures. The AUC of the normal cultured HHSteCs was $1.23 \times 10^{6}$ cells day $^{-1}$, whereas that of the senescent HHSteCs was $7.06 \times 10^{5}$ cells day $^{-1}$ (data not shown). Therefore, it was estimated that there were $2.5 \times 10^{3}$ particles produced cell ${ }^{-1}$ day $^{-1}$ for normal EVs and $4.2 \times 10^{3}$ particles cell ${ }^{-1}$ day $^{-1}$ for senescent EVs. Senescent HHSteCs released $\sim 1.7$-fold more EVs per cell than normal cultured HHSteCs, although the significance of this result could not be analyzed statistically.

Incorporation of EVs derived from HHSteCs into hepatoma cell lines. To confirm whether EVs secreted by HHSteCs were incorporated into both hepatoma cells and macrophages, EVs labeled with PKH67 were added to Hep3B cells and THP-1 

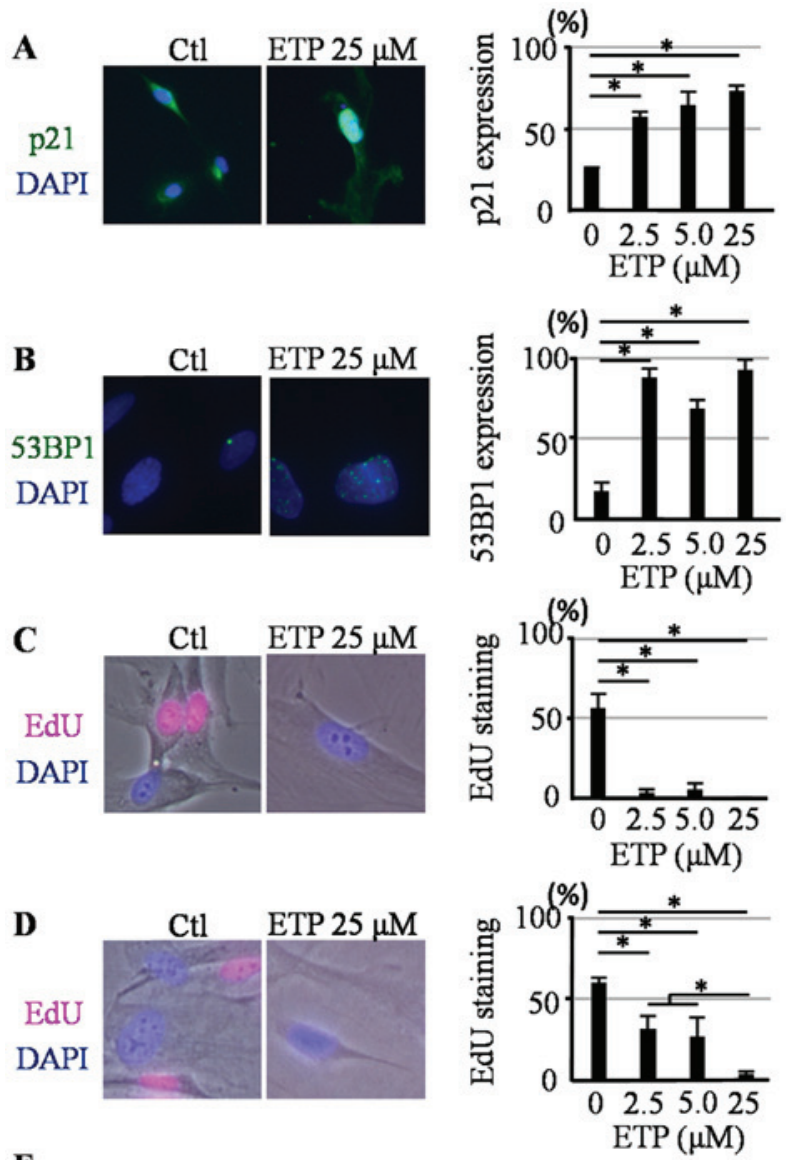

$\mathbf{E}$
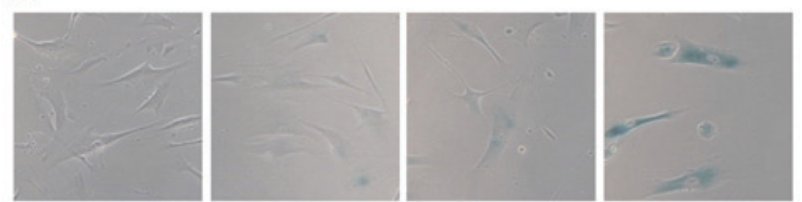

ETP $(-)$ (SA- $\beta-\operatorname{gal}(-))$

ETP (-)

ETP $5.0 \mu \mathrm{M} \quad$ ETP $25 \mu \mathrm{M}$

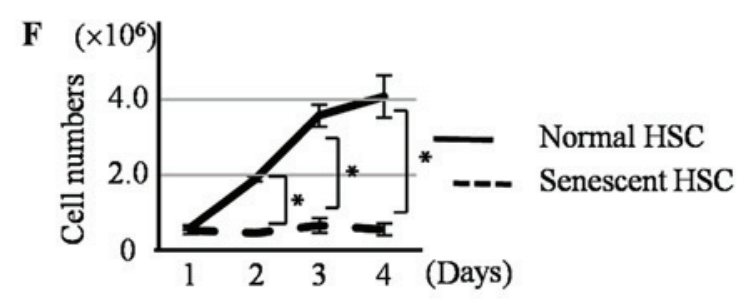

Figure 1. Induction of senescence in HHSteCs by ETP treatment. (A) p21 expression (green) was observed in HHSteCs treated with ETP at various concentrations. ETP treatment markedly upregulated p21 expression in HHSteCs compared with the control at all concentrations. (B) 53BP1 foci (green) were observed in HHSteCs treated with ETP at various concentrations. ETP treatment increased 53BP1 foci in HHSteCs compared to the control at all concentrations. (C) EdU uptake (pink) was analyzed in HHSteCs treated with ETP at various concentrations. ETP treatment dramatically reduced EdU uptake by HHSteCs compared with the control at all concentrations. (D) EdU uptake was analyzed in HHSteCs grown in fresh medium for 3 days following treatment with ETP at various concentrations. EdU uptake recovered in the HHSteCs treated with 2.5 and $5.0 \mu \mathrm{M}$ ETP, but not in HHSteCs treated with $25 \mu \mathrm{M}$ ETP. (E) SA- $\beta$-gal staining was observed in untreated HHSteCs and HHSteCs treated with 5.0 and $25 \mu \mathrm{M}$ ETP. Only HHSteCs treated with $25 \mu \mathrm{M}$ ETP exhibited a notable induction of senescence, as shown by the increase in staining. (F) Growth curves in fresh medium were compared between Normal HSC or Senescent HSCs for 3 days. No proliferation of senescent HSCs was observed in fresh medium. ${ }^{*} \mathrm{P}<0.05$. ETP, etoposide; HHSteCs, human hepatic stellate cells; Normal HSC, HHSteCs that had been cultured in normal medium; Senescent HSC, HHSteCs that had been cultured in medium containing $25 \mu \mathrm{M}$ ETP.

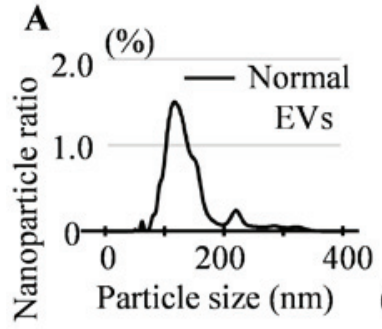

B
NC
(PBS)
NC
(unlabeled
EVs )

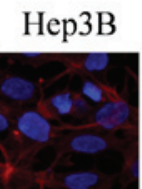

THP-1

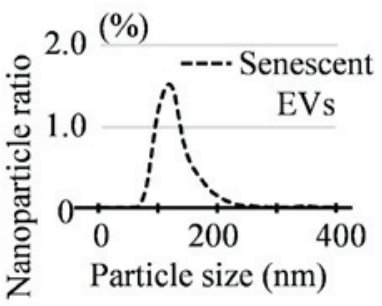

EVs $(+)$

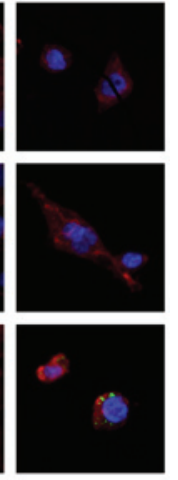

DAPI: blue Actin: red PKH67: green

Figure 2. Particle size of EVs secreted from HHSteCs and their incorporation into hepatoma cells. (A) Nanoparticle tracking analysis of normal and senescent EVs. Particle size distributions were nearly identical between normal and senescent EVs, and the majority of particles were $\sim 120 \mathrm{~nm}$ in size. (B) Hep3B cells and THP-1 cells were treated with PKH67-labelled EVs daily for 3 days and observed by fluorescence microscopy to examine EV incorporation. Magnification, x100. Unlabeled EVs and PBS without EVs (for confirmation of the absence of residual PKH67) were used as the NCs. PKH67 expression (green) was observed in only the Hep3B cells treated with PKH67-labeled EVs. EV, extracellular vesicle; NC, negative control; HHSteCs, human hepatic stellate cells; normal EVs, EVs derived from normal HHSteCs; senescent EVs, EVs derived from senescent HHSteCs.

cells daily for 3 days. PKH67 expression was observed in both cells (Fig. 2B), which suggests that EVs derived from HHSteCs were incorporated into cells.

Growth factor secretion from hepatoma cell lines and differentiated THP-1 cells treated with EVs derived from senescent HHSteCs. To assess the effect of senescent EVs on growth factor secretion from hepatoma cells, Hep3B and Huh7 cells were treated with either normal or senescent EVs daily for 3 days and the secretion of growth factors into the supernatant was measured using a panel of multiplex immunoassays. The difference in secretion of growth factors between normal and senescent EV treatments was <2-fold (Fig. S2). Several studies have shown that TAMs participate in hepatoma cell proliferation via changes in cytokine expression levels $(31,32)$. Thus, the effect of senescent EVs on growth factor secretion from THP-1 cells was assessed. Differentiated THP-1 cells were treated daily for 3 days with either normal or senescent EVs and growth factor secretion was measured. THP-1 cells treated with senescent EVs secreted significantly more EGF compared with those treated with normal EVs and the change was $>2$-fold (Fig. 3A).

EGF expression in THP-1 cells treated with EVs was further assessed by RT-qPCR and EGF protein-specific ELISA. The levels of EGF mRNA expression in THP-1 cells treated with senescent EVs was significantly higher compared with both the THP-1 cells treated with normal EVs and control (Fig. 3B). To further confirm the results obtained by multiplex immunoassays, EGF secretion was measured using ELISA. EGF secretion from THP-1 cells treated with senescent EVs was also increased compared with THP-1 cells treated with normal EVs and the control (Fig. 3C). 

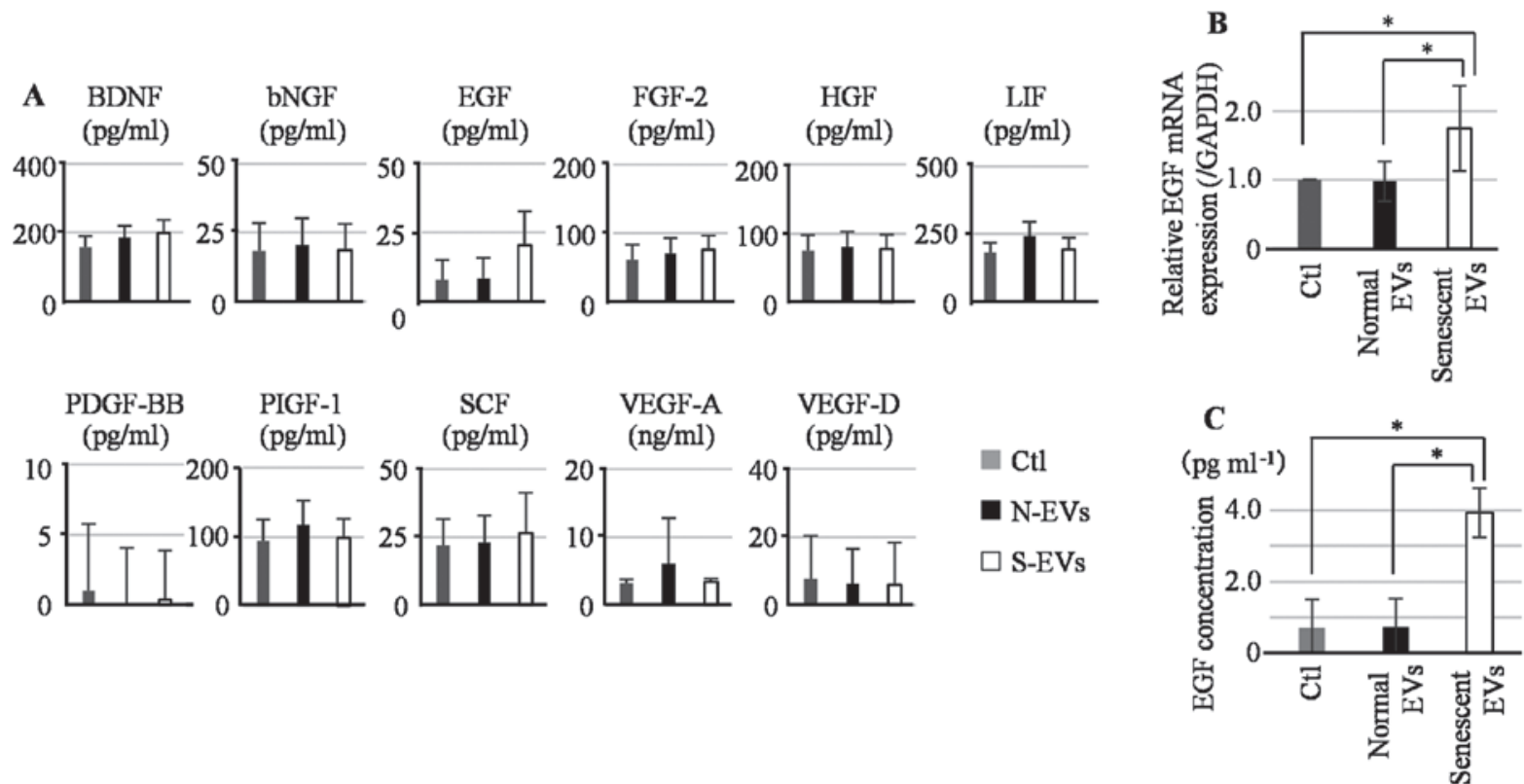

Figure 3. EGF upregulation in THP-1 cells treated with EVs secreted from senescent HHSteCs. (A) Comprehensive quantification of growth factors secreted by differentiated THP-1 cells treated with EVs was performed using multiplex immunoassays. THP-1 cells treated with senescent EVs secreted increased quantities of EGF compared with normal EVs, and the increase was >2-fold. (B) EGF mRNA expression levels were compared between the different treatments. THP-1 cells treated with senescent EVs had higher EGF mRNA expression levels compared with THP-1 cells treated with normal EVs and the control. (C) EGF secretion was examined in control THP-1 cells, THP-1 cells treated with normal EVs and THP-1 cells treated with senescent EVs. THP-1 cells treated with senescent EVs secreted significantly more EGF compared with the control or normal EV treated THP-1 cells. "P<0.05. EGF, epidermal growth factor; EV, extracellular vesicle; HHSteCs, human hepatic stellate cells; normal EVs, EVs derived from normal HHSteCs; senescent EVs, EVs derived from senescent HHSteCs.

A

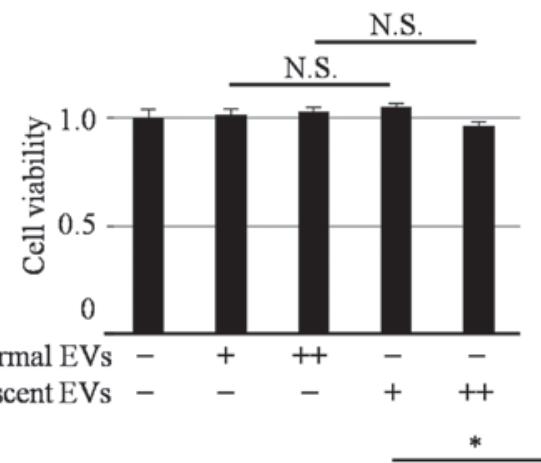

B

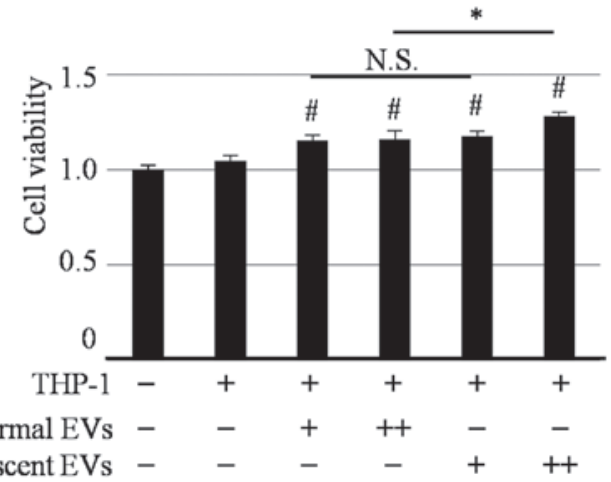

Figure 4. Impact of EVs on the proliferation of hepatoma cells alone or those co-cultured with THP-1 cells. To determine the effect of EVs on hepatoma cell proliferation, the viability of Hep3B cells treated with EVs was evaluated by MTS assays. (A) In the absence of THP-1 cells, neither normal EVs nor senescent EVs affected Hep3B cell viability. (B) Upon co-culture with THP-1 cells, both normal EVs and senescent EVs significantly increased Hep3B cell viability. Treatment with a higher quantity of senescent EVs significantly increased Hep3B cell viability compared with treatment with an equal quantity of normal EVs. ${ }^{*} \mathrm{P}<0.05 .{ }^{~} \mathrm{P}<0.05$ vs. co-culture with THP-1 cells without EV treatment N.S., not significant; EV, extracellular vesicle; normal EVs, EVs derived from normal human hepatic stellate cells; senescent EVs, EVs derived from senescent human hepatic stellate cells;,$+ 1 \times 10^{6}$ added daily;,$++ 3 \times 10^{6}$ added daily.
Effect of EVs derived from senescent HHSteCs on the proliferation of Hep3B cells. The effect of EVs derived from senescent HHSteCs on hepatoma cell viability was assessed. As shown in Fig. 4A, neither treatment with normal nor senescent EVs affected the proliferation of Hep3B cells. However, both EV treatments significantly increased the viability of Hep3B cells when co-cultured with differentiated THP-1 cells. Notably, this effect was significantly greater with senescent EVs compared with normal EVs (Fig. 4B). To validate the effect of EGF secretion from THP-1 cells on hepatoma cell lines, the cell viability of hepatoma cells in the presence or absence of erlotinib, an EGFR tyrosine kinase inhibitor, was determined. The concentration of erlotinib was set to $2.5 \mu \mathrm{M}$ to maintain Hep3B cell viability at $>80 \%$ (Fig. 5A). As shown in Fig. 5B, senescent EVs were more effective at enhancing Hep3B cell viability compared with EVs in the absence of erlotinib; however, this enhancing effect was inhibited in the presence of erlotinib. This suggests that the effect of senescent EVs on the proliferation of hepatoma cells co-cultured with THP-1 cells was dependent on EGF secreted from THP-1 cells.

\section{Discussion}

In the present study, it was demonstrated that senescence could be induced in HHSteCs by treatment with ETP, and that senescent HHSteCs released increased quantities of EV particles compared with normal HHSteCs. EVs derived from senescent HHSteCs resulted in increased EGF expression levels in THP-1 cells compared with EVs derived from normal HHSteCs, which promoted hepatoma cell viability. Therefore, EVs derived from senescent HSCs may create a 
A

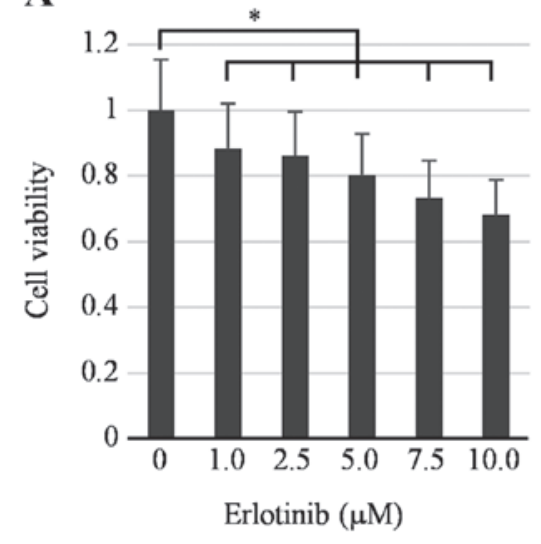

B

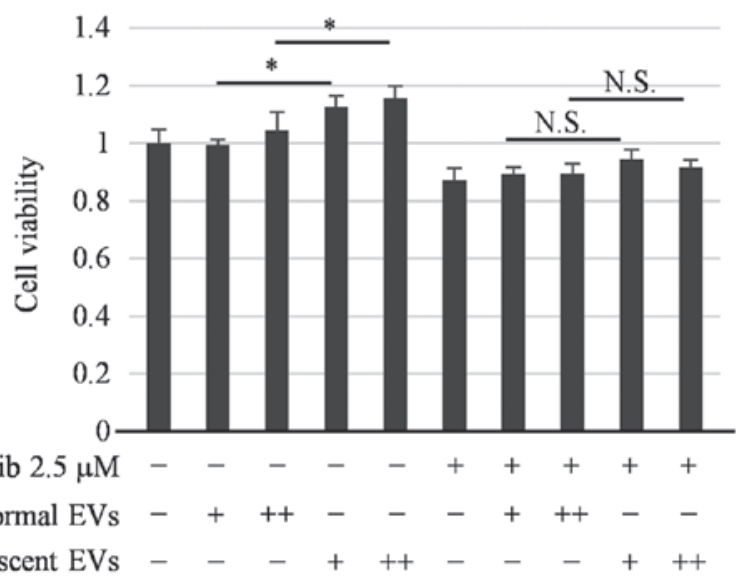

Figure 5. Effect of EVs derived from senescent HHSteCs co-cultured with THP-1 cells on the proliferation of hepatoma cells in the presence of erlotinib, an epidermal growth factor receptor inhibitor. To determine the involvement of EGF secreted from senescent HHSteCs in hepatoma cell proliferation upon co-culture with THP-1 cells, the viability of Hep3B cells co-cultured with THP-1 cells was evaluated in the absence or presence of erlotinib using MTS assays. (A) The effect of erlotinib on the inhibition of cell viability was examined at each concentration. Cell viability was maintained at $>80 \%$ with 1.0 and $2.5 \mu \mathrm{M}$ erlotinib treatment. (B) Cells were treated with two different quantities of EV particles daily for 3 days. Senescent EVs were more effective at enhancing Hep3B cell viability compared with normal EVs in the absence of erlotinib; however this effect was abrogated in the presence of erlotinib. ${ }^{\text {P }<0.05 . ~ N . S ., ~ n o t ~}$ significant; EV, extracellular vesicle; HHSteCs, human hepatic stellate cells; EGF, epidermal growth factor; +, $1 \times 10^{6}$ added daily; ++, $3 \times 10^{6}$ added daily; normal EVs, EVs derived from normal human hepatic stellate cells; senescent EVs, EVs derived from senescent human hepatic stellate cells.

more conducive tumor microenvironment for proliferation of hepatoma cells.

Senescent HSCs affect their surrounding cells, inducing alterations to the hepatic microenvironment. Krizhanovsky et al (33) examined the role of senescent HSCs in the hepatic microenvironment in detail. They showed that hepatic fibrosis develops in the absence of HSC senescence induction and that senescent HSCs promote the activity of NK cells to eliminate the activated HSCs which cause progression of hepatic fibrosis. However, they also hypothesized that senescent HSCs accumulate in the presence of sustainable and excessive liver damage, caused by viruses or hepatic steatosis, beyond the means of a physiological immune response. In addition, Yoshimoto et al (23) showed that senescent HSCs promote progression of liver cancer. At present, therapy targeting senescent cells has also been studied. Ogrodnik et al (34) reported that a combination of the selective senolytic agents dasatinib and quercetin reduced hepatic steatosis (34). Senescent cells are now regarded as attractive targets for novel therapeutic strategies. However, it is necessary to further elucidate their contributions to the tumor microenvironment.

To date, there have been numerous reports on the effects of EVs secreted by hepatocytes on stellate cells (35-37); however, to the best of our knowledge, there are no report on the effects of EVs secreted by stellate cells on hepatocytes or surrounding cells. In addition, it has been reported that the efficiency of EV uptake varies depending on the type of recipient cells (38). Accordingly, EV uptake by hepatoma cells and macrophage cells was initially determined and confirmed, and EVs affected the secretion of cytokines. Li et al (39) reported that EVs containing oncomiRs secreted from hepatoma cells are incorporated into HSCs, and that EVs secreted from HSCs promote HCC progression as a positive feedback mechanism. Furthermore, Wan et al (40) showed that exosomes secreted from activated HSCs are taken up by Kupffer cells, as well as the HSCs themselves, which increases the expression of
GLUT1 and PKM2. Therefore, EVs secreted by HSCs are actively taken up by surrounding cells including hepatocytes and macrophage cells with functional effects on the hepatic microenvironment.

Several studies have shown that the impact of EVs on surrounding cells varies by EV content and is regulated by cell conditions $(41,42)$. Regarding the effect of EVs derived from senescent cells on cancer cells, Takasugi et al (43) showed that EVs derived from senescent cells are absorbed by several breast cancer cell lines via EphA2, which resulted in SASP factor-like cancer progression. Similarly, in the present study, it was shown that EVs derived from senescent HSCs increased secretion of EGF from THP-1 cells, which in-turn promoted the proliferation of the hepatoma cells co-cultured with these cells. EVs derived from senescent HSCs may thus indirectly contribute to the formation of an environment conducive to the development of HCC.

There are some limitations to the present study. The primary limitation is that these results are based entirely on in vitro assays. Additionally, the number of EVs used for treatment was based on an approximate prediction method. The ratio of stellate cells in vivo is equivalent to $\sim 10 \%$ of the number of parenchymal cells $(21,44)$. Thus, the same ratio was used in the present study. As described in the results section, a single HHSteC secreted 2,500-4,000 particles per day. Therefore, the number of EVs used for treatment was set to $\sim 400 \mathrm{x}$ the number of hepatoma cells. However, it is unclear the number of EVs secreted from HSCs in the liver microenvironment in vivo. It is also possible that-various changes to the liver state may result in changes of the number of EVs secreted from HSCs. In addition, as the experiments in the present study were only performed in vitro, the conditions are considerably different from in vivo where immune cells are also involved. As reported by Krizhanovsky et al (33), senescent HSCs can modulate the immune system in vivo. Therefore, immunomodulatory signals from EVs derived from senescent HSCs 
should be explored in the future. However, the findings of the present study highlight the role of EVs derived from senescent HSCs in promoting tumor growth, similar to that observed with the SASP. Therefore, in our future studies, the effect of EVs derived from senescent HSCs in vivo will be assessed. In conclusion, senescent HSCs released increased quantities of EVs compared with normal HSCs. Similar to, SASP factors, EVs from senescent HSCs promote HCC development by upregulating EGF production in macrophages.

\section{Acknowledgements}

Not applicable.

\section{Funding}

No funding was received.

\section{Availability of data and materials}

The datasets used and/or analyzed during the present study are available from the corresponding author upon reasonable request.

\section{Authors' contributions}

All authors have contributed to this work and read and approved the manuscript. YM and SM designed the study, performed the experiments, wrote the article and interpreted the data. YK, SN, RS, MH and HS performed the experiments. TH, HM, NT and $\mathrm{KN}$ interpreted and analyzed the data. All authors have read and approved the final version of the manuscript.

\section{Ethics approval and consent to participate}

Not applicable.

\section{Patients consent for publication}

Not applicable.

\section{Competing interests}

The authors declare that they have no competing interests.

\section{References}

1. Forner A, Reig M and Bruix J: Hepatocellular carcinoma. Lancet 391: 1301-1314, 2018

2. Baecker A, Liu X, La Vecchia C and Zhang ZF: Worldwide incidence of hepatocellular carcinoma cases attributable to major risk factors. Eur J Cancer Prev 27: 205-212, 2018.

3. Torre LA, Bray F, Siegel RL, Ferlay J, Lortet-Tieulent J and Jemal A: Global cancer statistics, 2012. CA Cancer J Clin 65: 87-108, 2015

4. Katoh M: FGFR inhibitors: Effects on cancer cells, tumor microenvironment and whole-body homeostasis (Review). Int J Mol Med 38: 3-15, 2016.

5. Son B, Lee S, Youn H, Kim E, Kim W and Youn B: The role of tumor microenvironment in therapeutic resistance. Oncotarget 8 : 3933-3945, 2017.

6. Bissell MJ and Hines WC: Why don't we get more cancer? A proposed role of the microenvironment in restraining cancer progression. Nat Med 17: 320-329, 2011.
7. Maia J, Caja S, Strano Moraes MC, Couto N and Costa-Silva B: Exosome-based cell-cell communication in the tumor microenvironment. Front Cell Dev Biol 6: 18, 2018.

8. Rani B, Cao Y, Malfettone A, Tomuleasa C, Fabregat I and Giannelli G: Role of the tissue microenvironment as a therapeutic target in hepatocellular carcinoma. World J Gastroenterol 20: 4128-4140, 2014.

9. Tahmasebi Birgani M and Carloni V: Tumor microenvironment, a paradigm in hepatocellular carcinoma progression and therapy. Int J Mol Sci 18: E405, 2017.

10. Forbes SJ and Parola M: Liver fibrogenic cells. Best Pract Res Clin Gastroenterol 25: 207-217, 2011.

11. Kang N, Gores GJ and Shah VH: Hepatic stellate cells: Partners in crime for liver metastases? Hepatology 54: 707-713, 2011.

12. Ji J, Eggert T, Budhu A, Forgues M, Takai A, Dang H, Ye Q, Lee JS, Kim JH, Greten TF and Wang XW: Hepatic stellate cell and monocyte interaction contributes to poor prognosis in hepatocellular carcinoma. Hepatology 62: 481-495, 2015.

13. Liu Y and Cao X: The origin and function of tumor-associated macrophages. Cell Mol Immunol 12: 1-4, 2015.

14. Qian BZ and Pollard JW: Macrophage diversity enhances tumor progression and metastasis. Cell 141: 39-51, 2010.

15. Noy R and Pollard JW: Tumor-associated macrophages: From mechanisms to therapy. Immunity 41: 49-61, 2014.

16. Brown JM, Recht L and Strober S: The promise of targeting macrophages in cancer therapy. Clin Cancer Res 23: 3241-3250, 2017.

17. Dulai PS, Singh S, Patel J, Soni M, Prokop LJ, Younossi Z, Sebastiani G, Ekstedt M, Hagstrom H, Nasr P, et al: Increased risk of mortality by fibrosis stage in nonalcoholic fatty liver disease: Systematic review and meta-analysis. Hepatology 65: 1557-1565, 2017.

18. Liaw YF and Chu CM: Hepatitis B virus infection. Lancet 373: 582-592, 2009.

19. Fabregat I and Caballero-Diaz D: Transforming growth factor- $\beta$-induced cell plasticity in liver fibrosis and hepatocarcinogenesis. Front Oncol 8: 357, 2018.

20. Fabregat I, Moreno-Caceres J, Sanchez A, Dooley S, Dewidar B, Giannelli $\mathrm{G}$ and Ten Dijke P; IT-LIVER Consortium: TGF- $\beta$ signalling and liver disease. FEBS J 283: 2219-2232, 2016.

21. Yin C, Evason KJ, Asahina K and Stainier DY: Hepatic stellate cells in liver development, regeneration, and cancer. J Clin Invest 123: 1902-1910, 2013.

22. Thompson AI, Conroy KP and Henderson NC: Hepatic stellate cells: Central modulators of hepatic carcinogenesis. BMC Gastroenterol 15: 63, 2015.

23. Yoshimoto S, Loo TM, Atarashi K, Kanda H, Sato S, Oyadomari S, Iwakura Y, Oshima K, Morita H, Hattori M, et al: Obesity-induced gut microbial metabolite promotes liver cancer through senescence secretome. Nature 499: 97-101, 2013.

24. Wendler F, Favicchio R, Simon T, Alifrangis C, Stebbing J and Giamas G: Extracellular vesicles swarm the cancer microenvironment: From tumor-stroma communication to drug intervention. Oncogene 36: 877-884, 2017.

25. Borges FT, Reis LA and Schor N: Extracellular vesicles: Structure, function, and potential clinical uses in renal diseases. Braz J Med Biol Res 46: 824-830, 2013

26. Raposo G and Stoorvogel W: Extracellular vesicles: Exosomes, microvesicles, and friends. J Cell Biol 200: 373-383, 2013.

27. Akers JC, Gonda D, Kim R, Carter BS and Chen CC: Biogenesis of extracellular vesicles (EV): Exosomes, microvesicles, retroviruslike vesicles, and apoptotic bodies. J Neurooncol 113: 1-11, 2013.

28. Cheng L, Sharples RA, Scicluna BJ and Hill AF: Exosomes provide a protective and enriched source of miRNA for biomarker profiling compared to intracellular and cell-free blood. J Extracell Vesicles 3: 2014.

29. Cai S, Cheng X, Pan X and Li J: Emerging role of exosomes in liver physiology and pathology. Hepatol Res 47: 194-203, 2017.

30. Livak KJ and Schmittgen TD: Analysis of relative gene expression data using real-time quantitative PCR and the 2(-Delta Delta C(T)) method. Methods 25: 402-408, 2001.

31. Vansaun MN, Mendonsa AM and Lee Gorden D: Hepatocellular proliferation correlates with inflammatory cell and cytokine changes in a murine model of nonalchoholic fatty liver disease. PLoS One 8: e73054, 2013.

32. Shirabe K, Mano Y, Muto J, Matono R, Motomura T, Toshima T, Takeishi K, Uchiyama H, Yoshizumi T, Taketomi A, et al: Role of tumor-associated macrophages in the progression of hepatocellular carcinoma. Surg Today 42: 1-7, 2012. 
33. Krizhanovsky V, Yon M, Dickins RA, Hearn S, Simon J, Miething C, Yee H, Zender L and Lowe SW: Senescence of activated stellate cells limits liver fibrosis. Cell 134: 657-667, 2008.

34. Ogrodnik M, Miwa S, Tchkonia T, Tiniakos D, Wilson CL, Lahat A, Day CP, Burt A, Palmer A, Anstee QM, et al: Cellular senescence drives age-dependent hepatic steatosis. Nat Commun 8: 15691, 2017.

35. Povero D, Panera N, Eguchi A, Johnson CD, Papouchado BG, de Araujo Horcel L, Pinatel EM, Alisi A, Nobili V and Feldstein AE: Lipid-induced hepatocyte-derived extracellular vesicles regulate hepatic stellate cell via microRNAs targeting PPAR- $\gamma$. Cell Mol Gastroenterol Hepatol 1: 646-663 e4, 2015.

36. Devhare PB, Sasaki R, Shrivastava S, Di Bisceglie AM, Ray R and Ray RB: Exosome-mediated intercellular communication between hepatitis $C$ virus-infected hepatocytes and hepatic stellate cells. J Virol 91: e 02225-e02216, 2017.

37. Royo F, Schlangen K, Palomo L, Gonzalez E, Conde-Vancells J, Berisa A, Aransay AM and Falcon-Perez JM: Transcriptome of extracellular vesicles released by hepatocytes. PLoS One 8: e68693, 2013.

38. Feng D, Zhao WL, Ye YY, Bai XC, Liu RQ, Chang LF, Zhou Q and Sui SF: Cellular internalization of exosomes occurs through phagocytosis. Traffic 11: 675-687, 2010.

39. Li J, Yan Y, Ang L, Li X, Liu C, Sun B, Lin X, Peng Z, Zhang X, Zhang Q, et al: Extracellular vesicles-derived oncomirs mediate communication between cancer cells and cancer-associated hepatic stellate cells in hepatocellular carcinoma microenvironment. Carcinogenesis: May 29, 2019 (Epub ahead of print). doi: 10.1093/carcin/bgz096.
40. Wan L, Xia T, Du Y, Liu J, Xie Y, Zhang Y, Guan F, Wu J, Wang X and Shi C: Exosomes from activated hepatic stellate cells contain GLUT1 and PKM2: A role for exosomes in metabolic switch of liver nonparenchymal cells. FASEB J 33: 8530-8542, 2019.

41. Ota Y, Takahashi K, Otake S, Tamaki Y, Okada M, Aso K, Makino Y, Fujii S, Ota T and Haneda M: Extracellular vesicle-encapsulated miR-30e suppresses cholangiocarcinoma cell invasion and migration via inhibiting epithelial-mesenchymal transition. Oncotarget 9: 16400-16417, 2018.

42. Kogure T, Yan IK, Lin WL and Patel T: Extracellular vesicle-mediated transfer of a novel long noncoding RNA TUC339: A mechanism of intercellular signaling in human hepatocellular cancer. Genes Cancer 4: 261-272, 2013.

43. Takasugi M, Okada R, Takahashi A, Virya Chen D, Watanabe S and Hara E: Small extracellular vesicles secreted from senescent cells promote cancer cell proliferation through EphA2. Nat Commun 8: 15729, 2017.

44. Friedman SL: Hepatic stellate cells: Protean, multifunctional, and enigmatic cells of the liver. Physiol Rev 88: 125-172, 2008.

This work is licensed under a Creative Commons Attribution-NonCommercial-NoDerivatives 4.0 International (CC BY-NC-ND 4.0) License. 